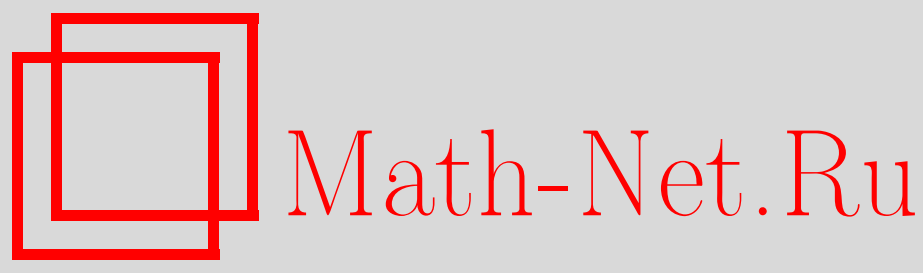

И. Кишимото, Т. Такахаши, Исследование структуры вакуума вблизи "единичных" решений, ТМФ, 2010, том 163, номер 3, 381-390

DOI: https://doi.org/10.4213/tmf6508

Использование Общероссийского математического портала Math-Net.Ru подразумевает, что вы прочитали и согласны с пользовательским соглашением http://www . mathnet.ru/rus/agreement

Параметры загрузки:

IP : 52.87 .193 .239

26 апреля 2023 г., 15:45:50

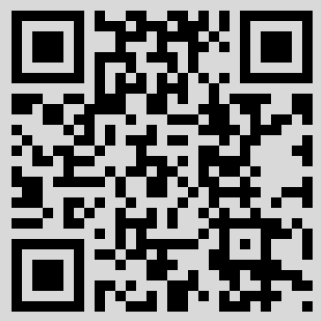




\title{
ФИЗИКА
}

Том 163, № 3

июнь, 2010

\section{ИССЛЕДОВАНИЕ СТРУКТУРЫ ВАКУУМА ВБЛИЗИ "ЕДИНИЧНЫХ" РЕШЕНИЙ}

\begin{abstract}
Исследована структура вакуума в теории поля открытых бозонных струн, разложенной вблизи решения, основанного на единичном струнном поле и параметризованного с помощью $a(\geqslant-1 / 2)$. Анализ разложенной теории вплоть до уровня 20 в приближении, основанном на отсечении уровней, показал, что при $a>-1 / 2$ эта теория имеет тахионное вакуумное решение. Кроме того, получено, что при $a=-1 / 2$ в разложенной теории существует неустойчивое вакуумное решение, и это решение, скорее всего, должно быть пертурбативным вакуумом открытых струн. Эти результаты разумно поддерживают гипотезу о том, что при $a>-1 / 2$ "единичное" решение является тривиальной чисто калибровочной конфигурацией, однако при $a=-1 / 2$ его можно рассматривать как тахионное вакуумное решение.
\end{abstract}

Ключевые слова: струнная теория поля, конденсация тахионов, единичное решение.

\section{1. ВВЕДЕНИЕ}

Струнная теория поля (СТП) открытых бозонных струн имеет классические решения, описывающие тахионный вакуум, в котором D-браны с присоединенными к ним открытыми струнами полностью аннигилируются. Численное решение для тахионного вакуума впервые было получено в рамках приближения, основанного на отсечении уровней, в калибровке Зигеля [1]. Затем было найдено аналитическое классическое решение Шнабля [2] и установлено, что оно обладает некоторыми свойствами тахионного вакуума. Вакуумная энергия для нетривиального аналитического решения точно сокращается с натяжением D-браны, и когомология кинетического оператора вблизи вакуума тривиальна [3].

В СТП имеются и аналитические решения другого типа, которые были получены ранее на основе единичного струнного поля вместо wedge-состояний, использованных в решении Шнабля [4] ${ }^{1)}$. Интересно, что рассмотренное в работах [4], [6]-[8]

${ }^{1)}$ В СТП имелись и другие попытки построения аналитических решений (см., например, работу [5] и приведенную там библиографию).

${ }^{*}$ Theoretical Physics Laboratory, RIKEN, Wako, Japan. E-mail: ikishimo@riken.jp

${ }^{\dagger}$ Department of Physics, Nara Women's University, Nara, Japan.

E-mail: tomo@asuka.phys.nara-wu.ac.jp 
"единичное" решение обладает некоторыми свойствами тахионного вакуума. Более того, это “единичное" решение позволяет понять существование замкнутых струн над тахионным вакуумом.

В разделе 2 мы кратко суммируем то, что нам известно о "единичном" решении. В разделах 3 и 4 будут представлены новые результаты для структуры вакуума вблизи этого решения [5]. Затем мы выскажем предположение о том, что это решение можно рассматривать как аналитическое решение для тахионного вакуума.

\section{2. РЕШЕНИЯ НА ОСНОВЕ ЕДИНИЧНОГО СТРУННОГО ПОЛЯ В ТЕОРИИ ПОЛЯ ОТКРЫТЫХ БОЗОННЫХ СТРУН}

"Единичное" решение можно записать как [4], [6]-[8]

$$
\Psi_{0}=Q_{\mathrm{L}}\left(e^{h}-1\right) I-C_{\mathrm{L}}\left((\partial h)^{2} e^{h}\right) I,
$$

где $I$ - единичное струнное поле, связанное с *-произведением, а полуструнные операторы $Q_{\mathrm{L}}$ и $C_{\mathrm{L}}$ определяются с использованием БРСТ-тока $J_{\mathrm{B}}(z)$ и духового поля $c(z)$ следующим образом:

$$
Q_{\mathrm{L}}(f)=\int_{C_{\mathrm{left}}} \frac{d z}{2 \pi i} f(z) J_{\mathrm{B}}(z), \quad C_{\mathrm{L}}(g)=\int_{C_{\mathrm{left}}} \frac{d z}{2 \pi i} g(z) c(z) .
$$

Здесь $C_{\text {left }}$ - контур вдоль единичной полуокружности, идущий от $-i$ до $i$, который по договоренности соответствует левой половине струны. Функция $h(z)$ определена на всей единичной окружности. Для функции $h(z)$, удовлетворяющей условиям $h(-1 / z)=h(z)$ и $h( \pm i)=0$, выполняются уравнения движения $Q_{\mathrm{B}} \Psi_{0}+\Psi_{0} * \Psi_{0}=0$.

Здесь и далее мы будем рассматривать "единичное" решение, полученное из функции

$$
\begin{aligned}
h(z) & =\ln \left(1+\frac{a}{2}\left(z+\frac{1}{z}\right)^{2}\right)= \\
& =-\ln (1-Z(a))^{2}-\sum_{n=1}^{\infty} \frac{(-1)^{n}}{n} Z(a)^{n}\left(z^{2 n}+z^{-2 n}\right),
\end{aligned}
$$

где $Z(a)=(1+a-\sqrt{1+2 a}) / a$. Для решения, удовлетворяющего условию вещественности, параметр $a \geqslant-1 / 2$. Приведенная функция представляет собой простейший пример, позволяющий выявить характерные черты “единичного” решения.

1. Оно имеет корректно определенное универсальное выражение в пространстве Фока: если мы разложим решение по состояниям в пространстве Фока,

$$
\left|\Psi_{0}(a)\right\rangle=\varphi_{0}(a) c_{1}|0\rangle+v_{0}(a) c_{1} L_{-2}^{X}|0\rangle+u_{0}(a) c_{-1}|0\rangle+\cdots
$$

то коэффициенты $\varphi_{0}(a), v_{0}(a)$ и $u_{0}(a)$ оказываются конечными для всех $a$. 
2. При $a>-1 / 2$ решение можно выразить в виде чистой калибровки, связанной с тривиальной конфигурацией; при $a=-1 / 2$ решение можно представить как некое сингулярное калибровочное преобразование тривиальной конфигурации [4]; для $a$ общего вида решение можно переписать как

$$
\Psi_{0}(a)=g(a) * Q_{\mathrm{B}} g^{-1}(a),
$$

но $g(a)$ становится сингулярным при $a=-1 / 2$.

Эти факты свидетельствуют о том, что при $a>-1 / 2$ решение является тривиальной чистой калибровкой, однако при $a=-1 / 2$ оно становится нетривиальной конфигурацией.

Более того, если мы разложим струнное поле как $\Psi=\Psi_{0}+\Phi$, то можно получить действие для флуктуации вблизи "единичного" решения. В разложенной теории кинетический оператор можно записать как

$$
\begin{aligned}
Q^{\prime}=(1 & +a) Q_{\mathrm{B}}+\frac{a}{2}\left(Q_{2}+Q_{-2}\right)+4 a Z(a) c_{0}-2 a Z(a)^{2}\left(c_{2}+c_{-2}\right)- \\
& -2 a\left(1-Z(a)^{2}\right) \sum_{n=2}^{\infty}(-1)^{n} Z(a)^{n-1}\left(c_{2 n}+c_{-2 n}\right),
\end{aligned}
$$

где мы разложили БРСТ-ток и духовое поле как $J_{\mathrm{B}}(z)=\sum_{n} Q_{n} z^{-n-1}$ и $c(z)=$ $\sum_{n} c_{n} z^{-n+1}$.

При $a>-1 / 2$ для разложенной теории были известны следующие факты:

1) действие, полученное при разложении вблизи решения, можно преобразовать обратно к действию с исходным БРСТ-зарядом [4];

2) новый БРСТ-заряд приводит к появлению когомологий, которые взаимно однозначно соответствуют когомологиям исходного БРСТ-заряда [6];

3) разложенная теория воспроизводит обычные амплитуды открытых струн [8].

Эти факты согласуются с гипотезой о том, что при $a>-1 / 2$ решение соответствует тривиальной чистой калибровке. С другой стороны, в теории, разложенной вблизи решения при $a=-1 / 2$, нами обнаружены совершенно другие свойства:

4) новый БРСТ-заряд имеет нулевую когомологию в гильбертовом пространстве с духовым числом 1 [6];

5) амплитуды рассеяния открытых струн обращаются в нуль, и результат согласуется с отсутствием возбуждений, представляющих открытые струны [8].

Исходя из этих фактов разумно ожидать, что "единичное" решение при $a=-1 / 2$ действительно соответствует тахионному вакуумному решению. Поэтому нам представляется, что это решение должно соответствовать тривиальной чистой калибровке почти для всей области параметров, а при $a=-1 / 2$ его можно рассматривать как тахионное вакуумное решение.

Для доказательства нашей гипотезы нужно непосредственно вычислить вакуумную энергию для "единичного" решения. Формально вакуумную энергию можно записать как

$$
V\left(\Psi_{0}(a)\right)=(\text { материальный сектор }) \times(\text { духовый сектор })=\infty \times 0,
$$


что представляет собой неопределенную величину. Для вычисления вакуумной энергии необходимо применить некоторую каноническую регуляризацию с целью разрешения неопределенности. Действительно, уровень можно рассматривать как параметр регуляризации при численном решении в калибровке Зигеля. Поэтому можно считать, что трудность в вычислении вакуумной энергии возникает из-за отсутствия подобного метода регуляризации для "единичного" решения.

Однако можно привести непрямые свидетельства в пользу того, что вакуумную энергию можно вычислить. Ранее нами была проанализирована структура вакуума в теории, разложенной вблизи "единичного" решения, в рамках приближения с отсеченными уровнями. При этом были получены следующие результаты:

6) численный анализ показывает, что непертурбативный вакуум, полученный при $a>-1 / 2$, исчезает, когда $а$ стремится к $-1 / 2[7]$;

7) при $a>-1 / 2$ при увеличении уровня отсечения энергия непертурбативного вакуума стремится к значению, близкому к величине, пригодной для сокращения натяжения D-браны [7].

Отсюда следует, что при $a>-1 / 2$ разложенная вблизи “единичного" решения теория имеет тахионый вакуум, но при $a=-1 / 2$ теория является устойчивой. Из соображений согласованности с теорией до разложения струнного поля следует, что при $a>-1 / 2$ энергия вакуума "единичного" решения равна нулю и равна энергии тахионного вакуума при $a=-1 / 2$.

\section{3. АННИГИЛЯЦИЯ ТАХИОННОГО ВАКУУМА}

Рассмотрим тахионный вакуум в теории, разложенной вблизи “единичного" решения. Разложенная теория обладает калибровочной симметрией относительно преобразований

$$
\delta \Phi=Q^{\prime} \Lambda+\Phi * \Lambda-\Lambda * \Phi
$$

где $Q^{\prime}$ определяется уравнением (6). Чтобы найти классические решения теории, наложим на флуктуации струнного поля условие калибровки Зигеля $b_{0} \Phi=0$. При наложении калибровки Зигеля потенциал можно записать как

$$
f_{a}(\Phi)=2 \pi^{2}\left(\frac{1}{2}\left\langle\Phi, c_{0} L(a) \Phi\right\rangle+\frac{1}{3}\langle\Phi, \Phi * \Phi\rangle\right),
$$

причем для тахионного вакуумного решения при $a=0$ он нормирован на -1 . Здесь оператор $L(a)$ имеет вид

$$
L(a)=(1+a) L_{0}+\frac{a}{2}\left(L_{2}+L_{-2}\right)+a\left(q_{2}-q_{-2}\right)+4(1+a-\sqrt{1+2 a}),
$$

где $L_{n}$ - полный генератор Вирасоро, а $q_{n}$ - мода духового тока ${ }^{2)}$.

2)Это выражение получено из выражения $L(a)=\left\{Q^{\prime}, b_{0}\right\}$. Его можно переписать, используя только твистованные духами операторы Вирасоро, как это было сделано в работе [8]. 


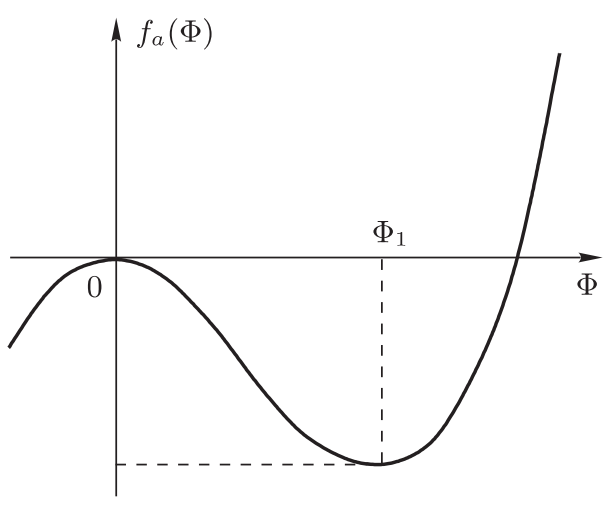

a

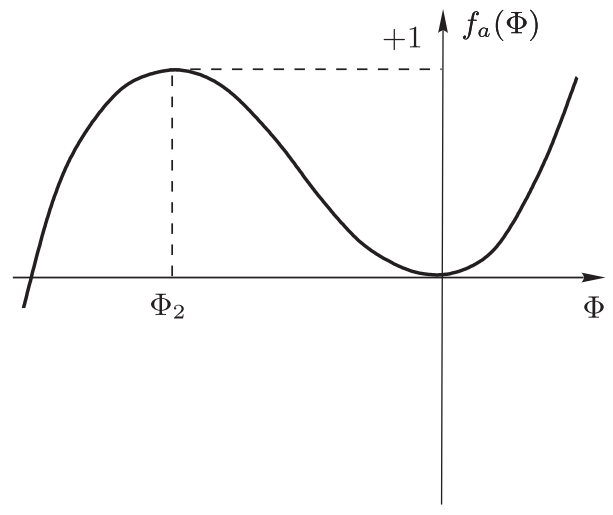

б

Рис. 1. Структура вакуума, ожидаемая для теории, разложенной вблизи "единичного" решения: а - при $a>-1 / 2$ теория должна иметь нетривиальное вакуумное решение, для которого энергия вакуума сокращает натяжение D-браны; б - при $a=-1 / 2$ тривиальная конфигурация $\Phi=0$ должна быть устойчивой; ожидается существование устойчивого решения, причем соответствующая ему энергия вакуума должна быть равна натяжению D-браны.

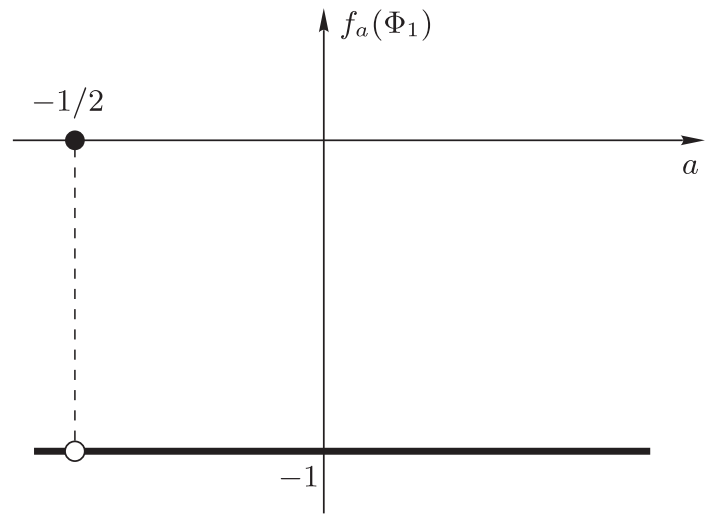

Рис. 2. Значения энергии вакуума, ожидаемые для нетривиального устойчивого решения $\Phi_{1}$.

Если "единичное" решение соответствует тривиальной чистой калибровке при $a>-1 / 2$ и тахионному вакууму при $a=-1 / 2$, то потенциал (9) должен иметь вид, приведенный на рис. 1 . При $a>-1 / 2$ конфигурация тахионного вакуума $\Phi_{1}$ должна минимизировать потенциал, поскольку для $\Phi$ разложенная теория все еще

2 Теоретическая и математическая физика, т. 163, № 3, 2010 г. 


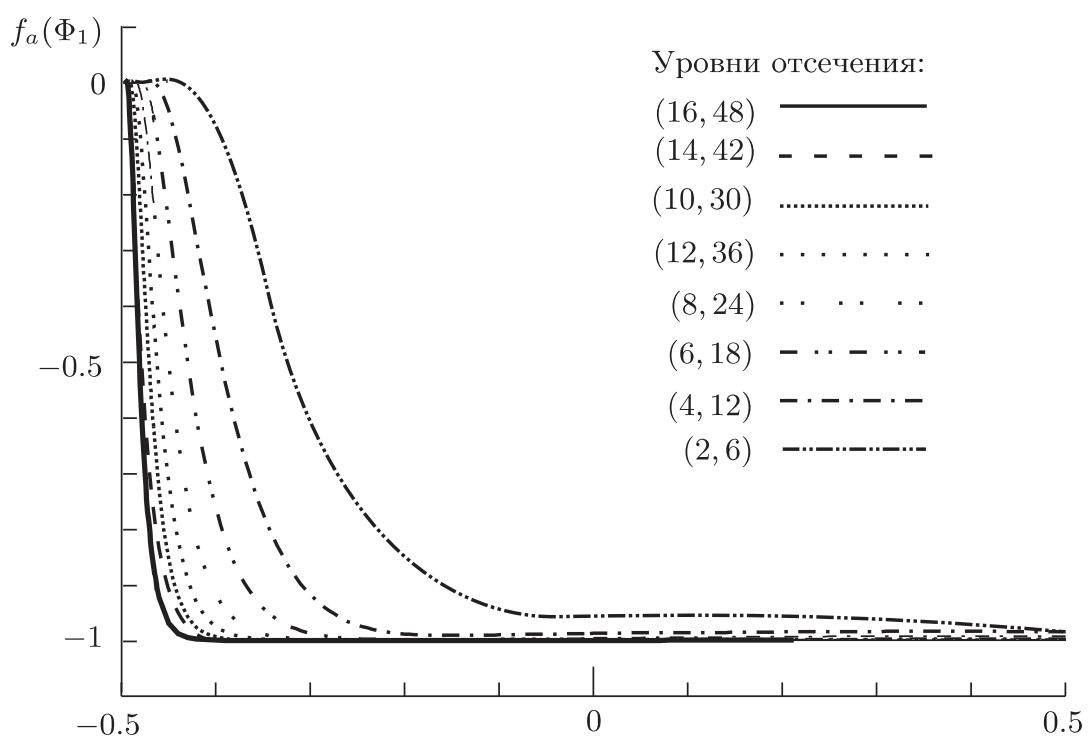

Рис. 3. Энергия вакуума для численных устойчивых решений в теории, разложенной вблизи "единичного" решения.

является теорией над пертурбативным вакуумом открытых струн. Однако при $a=-1 / 2$ тривиальная конфигурация $\Phi=0$ должна быть устойчивой, поскольку ожидается, что разложенная теория уже сформулирована над тахионным вакуумом. Кроме того, теория при $a=-1 / 2$ должна иметь неустойчивое решение, соответствующее пертурбативному вакууму открытых струн для неразложенной исходной теории.

Предположим, что мы получили тахионное вакуумное решение $\Phi_{1}$ в разложенной теории и вычислили соответствующую ему энергию вакуума. Тогда энергия вакуума $f_{a}\left(\Phi_{1}\right)$ задается как функция параметра $a$. Если потенциал имеет такую структуру, как предполагалось ранее, то при $a>-1 / 2$ энергия вакуума равна -1 , однако решение $\Phi_{1}$ становится тривиальным, и поэтому соответствующая ему энергия вакуума исчезает при $a=-1 / 2$ (см. рис. 2).

Для подтверждения высказанного предположения рассмотрим устойчивое решение $\Phi_{1}$, полученное в рамках приближения с отсечением уровней. Поскольку отсечение уровней представляет собой хорошую аппроксимацию, при увеличении уровня отсечения энергия вакуума для соответствующего решения должна стремиться к ступенчатой функции, как показано на рис. 2. Для нахождения устойчивого решения применим итерационный алгоритм, аналогично тому, как это было сделано в работах [9], [10].

На рис. 3 приведены значения вакуумной энергии для получающегося решения. Видно, что при увеличении уровня отсечения при различных значениях $a$ получающиеся значения стремятся к энергии тахионого вакуума, равной -1 . Далее, при 


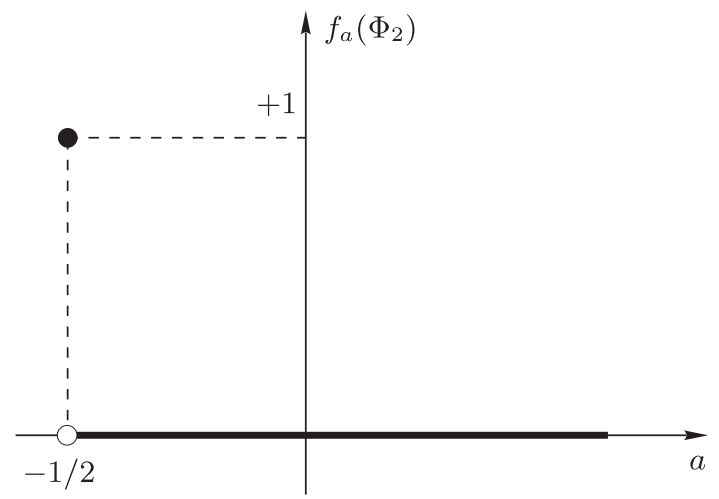

Рис. 4. Вакуумная энергия, ожидаемая для нетривиального устойчивого решения $\Phi_{2}$.

уменьшении $a$ до значения $-1 / 2$ энергия вакуума быстро возрастает от -1 до нуля. В целом при увеличении уровня отсечения значения стремятся к ступенчатой функции, как показано на рис. 2. Эти результаты подтверждают наше предположение о структуре вакуума, связанной с устойчивым решением $\Phi_{1}$.

\section{4. ВОЗНИКНОВЕНИЕ НЕУСТОЙЧИВОГО ВАКУУМА}

Рассмотрим неустойчивое решение в теории, разложенной вблизи "единичного" решения. При $a>-1 / 2$ разложенная теория неустойчива при $\Phi=0$. Однако ожидается, что при $a=-1 / 2$ разложенная теория относится уже к тахионному вакууму; а именно, $\Phi=0$ является устойчивым вакуумом при $a=-1 / 2$. Если так и есть, то при $a=-1 / 2$ разложенная теория должна иметь нетривиальное неустойчивое решение, соответствующее пертурбативному вакууму в исходной теории.

Поскольку неустойчивое решение $\Phi_{2}$ должно соответствовать пертурбативному вакууму открытых струн, энергия вакуума, соответствующего неустойчивому решению, должна быть равна натяжению D-браны (а не минус натяжению). Поэтому ожидается, что вакуумная энергия для $\Phi_{2}$ должна вести себя, как показано на рис. 4. Функция $f_{a}\left(\Phi_{2}\right)$ тривиальным образом равна нулю при $a>-1 / 2$, однако она должна возрастать до +1 при $a=-1 / 2$ из-за появления неустойчивого решения.

В таблице приведены значения энергии вакуума и калибровочно-инвариантного перекрытия для $\Phi_{2}$ при $a=-1 / 2$. Видно, что по мере увеличения уровня отсечения энергия вакуума приближается к ожидаемому значению, равному +1 . Энергия вакуума демонстрирует превышение приблизительно на $19 \%$ на уровне $(20,60)$ и приблизительно на $260 \%$ на уровне $(2,6)$. Более того, калибровочно-инвариантное перекрытие также приблизительно равно ожидаемому значению -1 . Эти результаты свидетельствуют, что приближение за счет отсечения уровней можно использовать также для анализа неустойчивого решения. Следовательно, имеется разумное 
ТАБлицА. Энергия вакуума и калибровочно-инвариантное перекрытие для неустойчивого решения $\Phi_{2}$ при $a=-1 / 2$.

\begin{tabular}{|c|c|c|}
\hline Уровни & Энергия вакуума & $\begin{array}{c}\text { Калибровочно-инвариантное } \\
\text { перекрытие }\end{array}$ \\
\hline$(0,0)$ & 2.3105795 & -1.0748441 \\
\hline$(2,6)$ & 2.5641847 & -1.0156983 \\
\hline$(4,12)$ & 1.6550774 & -0.9539832 \\
\hline$(6,18)$ & 1.6727496 & -0.9207572 \\
\hline$(8,24)$ & 1.4193393 & -0.9377548 \\
\hline$(10,30)$ & 1.4168893 & -0.9110994 \\
\hline$(12,36)$ & 1.3035715 & -0.9237917 \\
\hline$(14,42)$ & 1.2986472 & -0.9056729 \\
\hline$(16,48)$ & 1.2357748 & -0.9229035 \\
\hline$(18,54)$ & 1.2310583 & -0.9086563 \\
\hline$(20,60)$ & 1.1915648 & -0.9212376 \\
\hline
\end{tabular}

подтверждение того факта, что, как и ожидалось, неустойчивое решение действительно существует в разложенной теории при $a=-1 / 2$.

Рассмотрим неустойчивое решение $\Phi_{2}$ при различных $a(>-1 / 2)$. Получающаяся вакуумная энергия для неустойчивого решения показана на рис. 5. Значение энергии вакуума приблизительно равно ожидаемому значению при $a=-1 / 2$, однако при возрастании $a$ оно быстро убывает до нуля ${ }^{3)}$. Таким образом, из рис. 5 видно, что при увеличении уровня отсечения вакуумная энергия неустойчивого решения, как и ожидалось, стремится к ступенчатой функции.

\section{5. ЗАКЛЮЧЕНИЕ И ОБСУЖДЕНИЕ}

Мы нашли, что в калибровке Зигеля устойчивое и неустойчивое решения $\Phi_{1}$ и $\Phi_{2}$ численно существуют в теории, разложенной вблизи решения, основанного на единичном струнном поле. Для этих решений мы вычислили энергию вакуума в рамках приближения с отсечением уровней, действуя вплоть до уровня $(20,60)$. В нашей работе [5] мы также вычислили калибровочно-инвариантное перекрытие для этих решений. При увеличении уровня отсечения зависимость калибровочных инвариантов от параметра а замечательным образом стремится к поведению, ожидаемому исходя из структуры вакуума на рис. 1. Эти результаты в сильной степени поддерживают гипотезу о том, что при $a>-1 / 2$ "единичное" решение соответствует

\footnotetext{
3) Заметим, что на рис. 5 параметр а изменяется от -0.5 до -0.48 , и эта область изменения значительно у́же области изменения на рис. 3.
} 


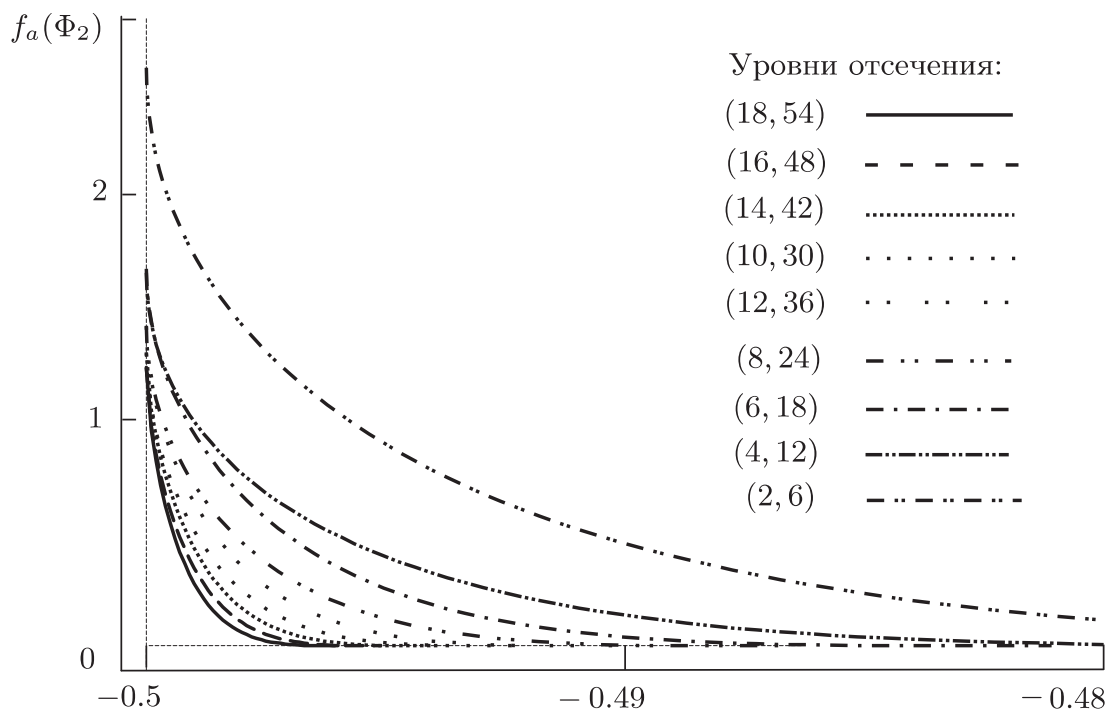

Рис. 5. Энергия вакуума для неустойчивого решения при различных $a$.

тривиальной чисто калибровочной конфигурации, однако при $a=-1 / 2$ его можно рассматривать как тахионное вакуумное решение.

Хотя приведенные результаты весьма обнадеживают, прямые вычисления остаются одной из наиболее сложных проблем в СТП. Однако успех численного анализа является характерной чертой “единичного” решения. В дополнение к решениям типа решения Шнабля “единичное” решение, как можно думать, предоставляет дополнительные подходы к углубленному пониманию СТП. В частности, мы ожидаем, что картина на мировом листе в разложенной теории прояснит существование замкнутых струн над тахионным вакуумом [11], [12].

Благодарности. Данная работа выполнена при частичной поддержке JSPS Grant-in-Aid for Scientific Research (C) (\#21540269). Работа И. Кишимото частично поддержана со стороны Special Postdoctoral Researchers Program at RIKEN. Работа T. Такахаши была частично поддержана за счет Nara Women's University Intramural Grant for Project Research. Численные расчеты в настоящей работе частично выполнялись с помощью Компьютерного центра Института теоретической физики Юкавы в университете Киото и в RIKEN Integrated Cluster of Clusters (RICC) facility.

\section{Список литературы}

[1] A. Sen, B. Zwiebach, JHEP, 03 (2000), 002; arXiv: hep-th/9912249.

[2] M. Schnabl, Adv. Theor. Math. Phys., 10:4 (2006), 433-501; arXiv: hep-th/0511286.

[3] I. Ellwood, M. Schnabl, JHEP, 02 (2007), 096; arXiv: hep-th/0606142.

[4] T. Takahashi, S. Tanimoto, JHEP, 03 (2002), 033; arXiv: hep-th/0202133.

[5] I. Kishimoto, T. Takahashi, Progr. Theoret. Phys., 122:2 (2009), 385-399; arXiv: 0904.1095. 
[6] I. Kishimoto, T. Takahashi, Progr. Theoret. Phys., 108:3 (2002), 591-602; arXiv: hep-th/0205275.

[7] T. Takahashi, Nucl. Phys. B, 670:1-2 (2003), 161-182; arXiv: hep-th/0302182.

[8] T. Takahashi, S. Zeze, Progr. Theoret. Phys., 110:1 (2003), 159-177; arXiv: hep-th/0304261.

[9] D. Gaiotto, L. Rastelli, JHEP, 08 (2003), 048; arXiv: hep-th/0211012.

[10] I. Kishimoto, T. Takahashi, Progr. Theoret. Phys., 121:4 (2009), 695-710; arXiv: 0902.0445.

[11] N. Drukker, JHEP, 08 (2003), 017; arXiv: hep-th/0301079.

[12] Y. Igarashi, K. Itoh, F. Katsumata, T. Takahashi, S. Zeze, Progr. Theoret. Phys., 114:6 (2005), 1269-1293; arXiv: hep-th/0506083. 